\title{
Element Specific Surface Reconstructions of Islands during Surfactant-Mediated Growth on Si (111)
}

\author{
A. Antons, ${ }^{1}$ K. Schroeder, ${ }^{1}$ B. Voigtländer, ${ }^{2}$ V. Cherepanov, ${ }^{2}$ R. Berger, ${ }^{1}$ and S. Blügel ${ }^{3}$ \\ ${ }^{1}$ Institut für Festkörperforschung, Forschungszentrum Jülich, D-52425 Jülich, Germany \\ ${ }^{2}$ Institut für Schichten und Grenzflächen, Forschungszentrum Jülich, D-52425 Jülich, Germany \\ ${ }^{3}$ Fachbereich Physik, Universität Osnabrück, D-49069 Osnabrück, Germany
}

(Received 30 April 2002; published 18 November 2002)

\begin{abstract}
The early stages of surfactant- (As, Sb) mediated homoepitaxial growth on $\mathrm{Si}$ (111) are examined by scanning tunneling microscopy and extensive ab initio calculations of $\operatorname{Si}_{n}$ clusters $(n \leq 12)$. The results reveal the different microscopic behavior of the two surfactants: On As-covered Si (111), one exclusively finds two-dimensional islands with double-layer height which show the $(1 \times 1)$ terrace structure. On Sb-covered $\mathrm{Si}$ (111), the islands show two different reconstructions: at the rim of the islands a $(1 \times 1)$ structure appears, while in the center the $(\sqrt{3} \times \sqrt{3})$ terrace structure is observed.
\end{abstract}

\section{DOI: $10.1103 /$ PhysRevLett.89.236101}

Surfactants (e.g., As or $\mathrm{Sb}$ and more recently $\mathrm{Bi}$ ) are used to modify the growth mode in heteroepitaxy of systems with large lattice mismatch [1-4]. For the $\mathrm{Si} / \mathrm{Ge}$ system thick layers of Ge grow in the layer-by-layer mode on $\mathrm{Sb}$ - or As-covered $\mathrm{Si}$ (111), whereas three-dimensional islands develop on the clean surface (Stranski-Krastanov mode). During epitaxy the surfactants float on top of the growing films and any deposited atom eventually has to be incorporated underneath the surfactant layer. In the past, two limiting cases have been investigated: the structure of full layers and the kinetics of single adatoms [510]. It was found that surfactants reduce the surface free energy by saturating Si dangling bonds, and govern the kinetics of single adatoms by controlling the competition between diffusion on top of the surfactant layer and the incorporation into the layer by exchange with a surfactant atom. But how growth proceeds after single atom exchange remains unknown to date. One has to address the intermediate stages, i.e., the formation of clusters with local changes of the surface structure. One example is the development and growth of the double-layer structure characteristic of the (111) surface.

This Letter deals exactly with these points. We show how the surfactant elements As and Sb act differently in $\mathrm{Si}$ (111) homoepitaxy. By scanning tunneling microscopy $(\mathrm{STM})$ we found that islands show only the $(1 \times 1)$ surface structure for As as surfactant, whereas islands show an alternating sequence of $(\sqrt{3} \times \sqrt{3}) \rightarrow(1 \times 1) \rightarrow$ $(\sqrt{3} \times \sqrt{3})$ surface structure for $\mathrm{Sb}$ as surfactant. We present extensive $a b$ initio calculations of the structures and energies of $\mathrm{Si}_{n}$ clusters which are able to explain the double-layer growth of Si on the As-terminated surface as well as the observed alternating sequence of surface reconstructions on the Sb-terminated surface.

The surfactant-covered surfaces were prepared by deposition of the saturation coverage of 1 monolayer (ML) of As or $\mathrm{Sb}$ at $700^{\circ} \mathrm{C}$ and $670^{\circ} \mathrm{C}$, respectively [5]. Figure 1 shows STM images of two-dimensional Si islands grown on the surfactant terminated surfaces. For As as surfac-
PACS numbers: 68.35.-p, 68.37.Ef, 68.43.Bc, 68.55.-a

tant [Figs. 1(a) and 1(c)] only steps with the height of a double layer are found. The surface on top of the islands as well as on the terraces always shows the $(1 \times 1)$ structure. On the other hand, for $\mathrm{Sb}$ as surfactant a $(\sqrt{3} \times \sqrt{3})$ reconstruction is found on the terraces and on top of the double-layer high two-dimensional islands [Figs. 1(b) and 1(d)]. At the rim of the islands a lower lying flat area is observed. We assign this structure to a $(1 \times 1)$ structure with $\mathrm{Sb}$ incorporated in the upper half of a double layer. The atoms are spaced so closely that atomic resolution is rarely obtained. The step from the terrace to the $(1 \times 1)$ rim appears to be a bit smaller than half of a double layer.
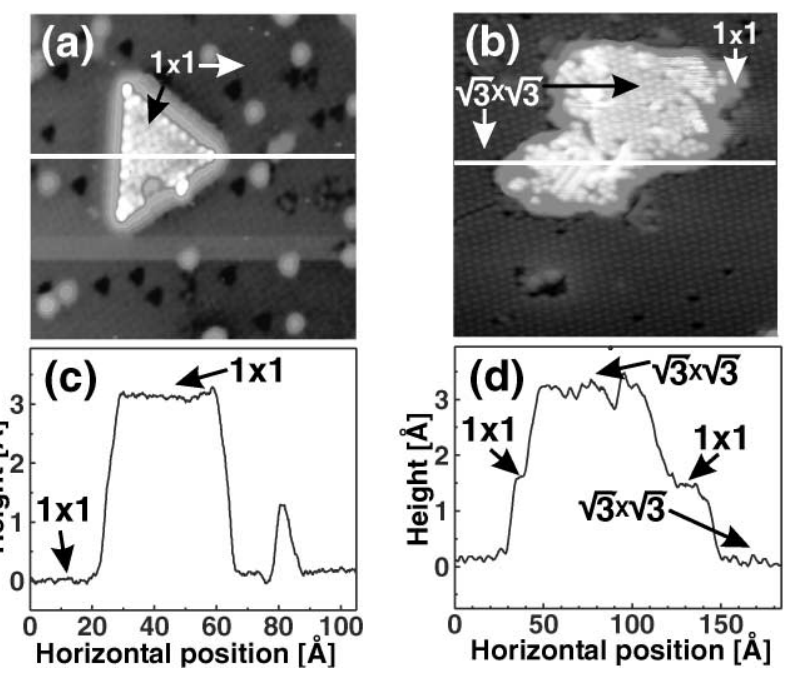

FIG. 1. STM images of surfactant covered $\mathrm{Si}$ islands on $\mathrm{Si}$ (111): (a) with As-coverage double layer height islands are formed with the same $(1 \times 1)$ structure as visible on the terrace; (b) with $\mathrm{Sb}$ coverage the islands have a outer rim of $(1 \times 1)$ structure and a center area which is $(\sqrt{3} \times \sqrt{3})$ reconstructed. [Image width and sample bias: $104 \AA, 1.9 \mathrm{~V}$ and $184 \AA,-1.75 \mathrm{~V}$ in (a) and (b), respectively]. In (c) and (d) line scans along the white lines in (a) and (b) are shown, respectively. 
The two observed structures of the $\mathrm{Si}$ (111) surfaces covered with $1 \mathrm{ML}$ of surfactant (As, $\mathrm{Sb}$ ) are shown in Fig. 2. In the (111) direction the diamond structure of $\mathrm{Si}$ exhibits a sequence of double layers separated by a nearest-neighbor $(\mathrm{NN})$ distance. The two layers within a double layer are separated by $1 / 3$ of a NN distance. In the $(1 \times 1)$ structure the surfactant atoms are sitting substitutionally in the upper layer of the terminating double layer [Fig. 2(a)]. In the $(\sqrt{3} \times \sqrt{3})$ structure the surfactant atoms sit on top of a complete Si double layer, forming T4-centered trimers [Fig. 2(b)]. These structures were found to be the equilibrium structures for $1 \mathrm{ML}$ As and $\mathrm{Sb}$, respectively, on $\mathrm{Si}(111)$ at room temperature [11,12], and also the most stable structure by ab initio calculations [6]. Both structures saturate all Si dangling bonds and show threefold coordination of the group- $\mathrm{V}$ elements, leaving one doubly occupied dangling bond on each group-V atom. With respect to the full double layer there are two significantly different symmetric adsorption sites: H3 sites above the middle of the sixfold rings and $\mathrm{T} 4$ sites above the atoms of the lower half layer.

In order to understand the experimentally observed growth behavior the following questions have to be addressed: (i) Why is double-layer growth favored compared to single-layer growth on $\operatorname{Si}(111)$ : $\operatorname{As}(1 \times 1)$; at what cluster size does it start? (ii) Why is double-layer growth not possible on $\mathrm{Si}(111): \mathrm{Sb}(1 \times 1)$; when does the cluster sequence start to deviate from that on $\mathrm{Si}(111)$ :As? (iii) Why is double-layer growth not possible on $\mathrm{Si}(111): \mathrm{Sb}(\sqrt{3} \times \sqrt{3})$; what determines the minimum size of the $(1 \times 1)$ islands before second-layer Sb trimers can start to grow (geometry, energy, or kinetics)?

We have calculated the relevant structures and energies which govern the Si cluster growth on surfactant-covered Si (111) using ab initio density functional theory. The calculations have been carried out with the ESTCOMPP code (Electronic Structure Code for Materials Properties and Processes, Forschungszentrum Jülich, 1999) [13], a parallelized ab initio molecular dynamics program in the spirit of Car and Parrinello [14]. It is based on the local density approximation (LDA) [15] to the density functional theory (DFT) [16], norm-conserving
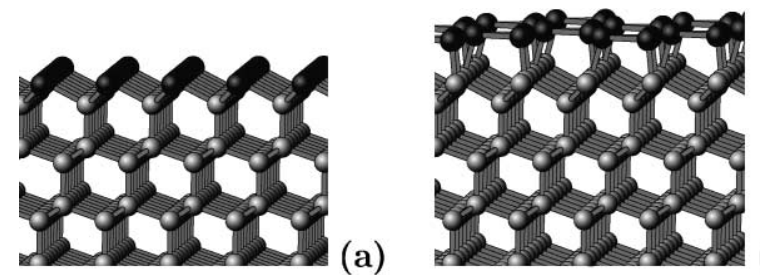

(b)

FIG. 2. Equilibrium surface structures of surfactant-covered $\mathrm{Si}$ (111) (light spheres: $\mathrm{Si}$, dark spheres: As, Sb): (a) the $(1 \times 1)$ structure, the $\mathrm{As}, \mathrm{Sb}$ atoms constitute the upper layer of the terminating double layer; (b) the $(\sqrt{3} \times \sqrt{3})$ structure, the As, $\mathrm{Sb}$ atoms form T4-centered trimers on top of a full Si double layer. pseudopotentials [17] of the Kleinman-Bylander form [18], a plane-wave basis set for the wave functions, and iterative diagonalization schemes $[19,20]$ for the eigenvalue problem. Force calculations combined with molecular relaxation are used to determine the minimum energy configurations and the reaction pathways [21]. The surfactant-covered surface is simulated by a repeated slab model consisting of six atomic $\mathrm{Si}$ (111) layers for the $(1 \times 1)$ surface structures in an inversion symmetric arrangement [and eight layers for the $(\sqrt{3} \times \sqrt{3})$ structures], $1 \mathrm{ML}$ surfactant on each $\mathrm{Si}$ surface, and one adatom or adatom cluster, respectively, on each surfactant layer. The surfaces were separated by a vacuum equivalent to eight atomic $\mathrm{Si}$ layers. The adatom clusters on $\mathrm{Si}(111)$ :As were modeled using a $(4 \times 4)$ lateral cell, and for $\mathrm{Si}(111): \mathrm{Sb}$ we used a $(3 \sqrt{3} \times 3 \sqrt{3})$ lateral cell with up to 294 atoms in the supercell because of the larger cluster sizes considered. We used four $\mathbf{k}_{\|}$points in the surface Brillouin zone and a cutoff energy of $13.69 \mathrm{Ry}$ for the plane-wave basis set [22]. The symmetry-unrestricted geometry optimization includes all atoms except those of the two innermost atomic layers of the slab. To establish minimum energy configurations we converged the forces acting on the atoms to less than $0.1 \mathrm{mRy} / \mathrm{a}$.u.

We have investigated the structures and energies of small surfactant-covered $\mathrm{Si}_{n}$ clusters of substitutionally incorporated $\mathrm{Si}$ atoms for $n \leq 4$ on the $(1 \times 1)$ surfaces and $n \leq 12$ on the $(\sqrt{3} \times \sqrt{3}) \mathrm{Si}(111): \mathrm{Sb}$ surface. No additional surfactant atoms were taken into account to decorate the clusters. For each cluster size we constructed several starting configurations with the condition that $\mathrm{Si}$ dangling bonds were avoided if possible. The final configurations were obtained by free relaxation of the initial structures. In total we investigated more than 50 different clusters. The binding energies for the most stable $\mathrm{Si}_{n}$ clusters on the $(\mathrm{As}, \mathrm{Sb})$-covered $(1 \times 1)$ surface are plotted versus the cluster size in Fig. 3. The atomic configurations are shown as insets. In the following we discuss the growth sequence of $\mathrm{Si}$ clusters first on the As and Sb-terminated $(1 \times 1)$ surfaces, and then on the Sb-terminated $(\sqrt{3} \times \sqrt{3})$ surface.

(i) $\mathrm{On} \mathrm{Si}(111): \mathrm{As}(1 \times 1)$ we find that the most stable structures are compact clusters whose energies decrease monotonically with the number of incorporated $\mathrm{Si}$ atoms. Generally, the shown clusters are stabilized by the saturation of bonds. For all clusters in which the $\mathrm{Si}$ atoms sit on sites with a tetrahedral neighborhood the binding energy is particularly large. The most stable Si dimer shown consists of two neighboring substitutional $\mathrm{Si}$ atoms, and the replaced $\mathrm{As}$ atoms form an $\mathrm{As}_{2}$ bridge between the $\mathrm{Si}$ atoms. In the stable $\mathrm{Si}$ trimer the three incorporated $\mathrm{Si}$ atoms are located at neighboring sites in the As layer, constituting a triangle which locally completes a Si double layer, and the three replaced As atoms form a T4-centered trimer on top of the Si atoms. In the most stable $\mathrm{Si}_{4}$ cluster the fourth $\mathrm{Si}$ atom replaces one of the As atoms of the trimer and lifts it to a nearly ideal 


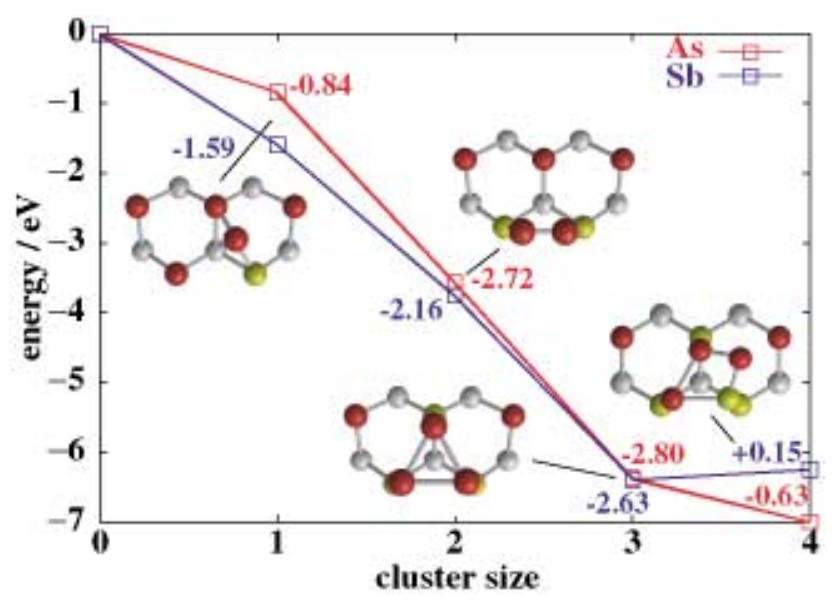

FIG. 3 (color). Development of small $\mathrm{Si}_{n}$ clusters (yellow balls) for $n=1-4$ on (As, Sb)-covered $\mathrm{Si}(111)(1 \times 1)$ during homoepitaxy (Si: gray balls; As, Sb: red balls). Plotted are the calculated energies $E_{c l}^{n}-n E(\mathrm{SiH} 3)$ which are gained by forming a $\mathrm{Si}_{n}$ cluster from $n$ single $\mathrm{Si}$ adatoms in the $\mathrm{H} 3$ position on top of the surfactant layer. The listed energies (in $\mathrm{eV}$ ) are the differences $\Delta E^{(n)}=E_{c l}^{(n)}-E_{c l}^{(n-1)}-E^{(1)}$ gained by forming a $\mathrm{Si}_{n}$ cluster from a $\mathrm{Si}_{n}-1$ cluster plus a single incorporated $\mathrm{Si}$ atom. $\mathrm{Si}(111)$ :As (red lines): The energies monotonically decrease up to $n=4$, the 4 th $\mathrm{Si}$ atom is attached to the As trimer. $\mathrm{Si}(111): \mathrm{Sb}$ (blue lines): The energies decrease up to $n=3$, but $\Delta E^{(4)}$ is positive. The forth $\mathrm{Si}$ atom is not attached to the $\mathrm{Sb}$ trimer.

position in the upper layer of the next double layer above the terrace, while the fourth $\mathrm{Si}$ atom sits in the lower layer. Although a Si dangling bond appears, this attachment is energetically favorable compared to an isolated $\mathrm{Si}$-As exchange on the As-covered terrace. Thus, this cluster of four exchanged Si atoms (containing three As atoms) is the nucleus for the double layer islands observed in experiments. Our proposal is that the growth beyond the tetramer proceeds by replacing the remaining two As atoms at the corners of the trimer with $\mathrm{Si}$ atoms, lifting these As atoms also up to the next higher layer. To saturate the Si dangling bonds additional As atoms are required. To avoid burying As atoms below the growing cluster further growth has to proceed by increasing the base of the cluster: $\mathrm{Si}$ adatoms are incorporated on the terrace by exchange with the As atoms next to the existing cluster.

(ii) On $\mathrm{Si}(111): \mathrm{Sb}(1 \times 1)$ the $\mathrm{Si}$ cluster formation up to $n=3$ follows the same sequence as for As coverage. But then, the fourth $\mathrm{Si}$ atom is more favorably exchanged on the Sb-covered terrace rather than attached to the existing $\mathrm{Sb}$ trimer. It serves as a nucleation center for a new $\mathrm{Sb}$ trimer on top of a complete $\mathrm{Si}$ double layer. As an important consequence double-layer growth on $\mathrm{Si}(111): \mathrm{Sb}(1 \times 1)$ cannot proceed, in contrast to the Asterminated surface. According to our calculations the energy of a single $\mathrm{Sb}$ trimer on a complete $\mathrm{Si}$ double layer is not very different from the energy per Sb trimer in the $\mathrm{Si}(111)$ :Sb surface with $(\sqrt{3} \times \sqrt{3})$ structure. Thus, we expect a structure of scattered $\mathrm{Sb}$ trimers on top of the
$(1 \times 1)$ islands as is experimentally observed at low growth temperatures $\left(T \approx 500^{\circ} \mathrm{C}\right)$. At the growth temperature of $670^{\circ} \mathrm{C}$, for which the STM image is shown in Fig. 1, the Sb trimers form a compact $(\sqrt{3} \times \sqrt{3})$ structure.

In our opinion, the different clustering behavior on As and Sb-covered Si (111) has the same origin as the different equilibrium surface structures. It is due to a subtle balance between the bond strengths of surfactantsurfactant, surfactant-Si and $\mathrm{Si}-\mathrm{Si}$ bonds in a given geometry. The decisive parameter is the covalent radius of the surfactant atoms which determines the necessary bond angle adjustment and local strain.

(iii) To study the growth sequence on the $\mathrm{Si}(111): \mathrm{Sb}(\sqrt{3} \times \sqrt{3})$ terrace structure we have calculated the structures and energies of $(1 \times 1) \mathrm{Si}_{n}$ clusters containing up to 12 additional substitutionally incorporated $\mathrm{Si}$ atoms. Because of the openness of the $(\sqrt{3} \times \sqrt{3})$ structure there are several possibilities how the single layer $(1 \times 1)$ clusters could grow. In addition, the incorporation of $\mathrm{Si}$ adatoms in the second layer on top of the $(1 \times 1)$ clusters is addressed, which leads to the nucleation of $\mathrm{Sb}$ trimers on a locally complete $\mathrm{Si}$ double layer above the terrace and the development of a new $(\sqrt{3} \times \sqrt{3})$ surface. The results are summarized in Fig. 4 which shows the $(1 \times 1) \mathrm{Si}_{12}$ cluster as an inset. The numbers indicate the

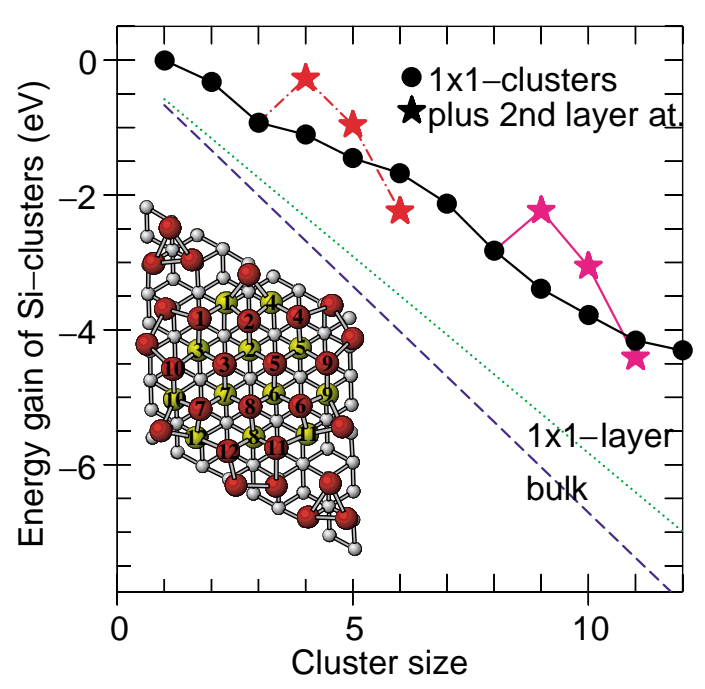

FIG. 4 (color). Development of small $\mathrm{Si}_{n}$ clusters $(n=1-12)$ on Sb-covered Si (111) $(\sqrt{3} \times \sqrt{3})$ during homoepitaxy. Plotted are the calculated energies (full points) $E_{c l}^{n}-n E_{B}$ gained by forming an $\mathrm{Sb}$-covered $\mathrm{Si}_{n}$ cluster with $(1 \times 1)$ surface structure from $n$ single incorporated $\mathrm{Si}$ atoms. The dot-dashed lines connect the calculated energies (full stars) when additional (up to 3 ) $\mathrm{Si}$ atoms are built into the second layer: red: on top of a $\mathrm{Si}_{3}$ cluster; magenta: on top of a $\mathrm{Si}_{8}$ cluster. The dotted lines are the energies obtained when each atom in a cluster would gain the energy per atom of a full $(1 \times 1)$ layer on the $(\sqrt{3} \times \sqrt{3})$ $\mathrm{Si}(111)$ :Sb surface (green), and of the Si bulk (blue), respectively. The inset shows the structure of the $\mathrm{Si}_{12}(1 \times 1)$ cluster (numbered atoms, yellow $\mathrm{Si}$, red $\mathrm{Sb}$ ). The numbers indicate the sequence of attachment. 
attachment sequence of the $\mathrm{Si}$ adatoms (yellow) which have replaced the $\mathrm{Sb}$ atoms (red) with the corresponding numbers by an exchange reaction. Taking into account the lowest energy configurations of the clusters we find that growth on the $(\sqrt{3} \times \sqrt{3})$ surface proceeds as follows: (a) The replaced $\mathrm{Sb}$ atoms sit on an $\mathrm{H} 3$ site above the trimer layer and always bind to the incorporated $\mathrm{Si}$ atom and to the two atoms forming the edge of the adjacent trimer, irrespective of the type of atoms sitting there ( $\mathrm{Si}$ or $\mathrm{Sb}$ ). Already one incorporated $\mathrm{Si}$ atom establishes an element of a $(1 \times 1) \mathrm{Sb}$-covered double layer [23]. This can, e.g., be seen in the inset of Fig. 4 for the Si-Sb pairs numbered 1 and 2 , respectively. (b) Threefold rings centered at the empty T4 sites of the $(\sqrt{3} \times \sqrt{3})$ structure (e.g., pairs 1-3) are completed to maximize the total number of bonds of $\mathrm{Sb}$ atoms in the upper layer to $\mathrm{Si}$ atoms in the lower layer and vice versa. (c) To incorporate $\mathrm{Si}$ atoms in the second layer above the terrace a nucleation barrier of the order of $1.0 \mathrm{eV}$ [see Fig. 4, energy difference for incorporating the first $\mathrm{Si}$ in the second layer rather than enlarging the $(1 \times 1)$ cluster by one] has to be overcome. But, the calculated energies for forming a complete $\mathrm{Sb}$ trimer in the second layer on a $(1 \times 1) \mathrm{Si}_{n}$ cluster [establishing the nucleus for double-layer growth on the $(\sqrt{3} \times \sqrt{3})$ terrace] are lower by about $0.5 \mathrm{eV}$ compared to the energies of the $\mathrm{Si}_{n}+3$ cluster with $(1 \times 1)$ structure. This is demonstrated in Fig. 4, where the energies of incorporating 1,2, and $3 \mathrm{Si}$ atoms in the second layer (stars) are compared to the energies gained by increasing the $(1 \times 1)$ cluster, starting from a $\mathrm{Si}_{3}$ cluster and a $\mathrm{Si}_{8}$ cluster, respectively. For the $\mathrm{Si}_{3}$ cluster the $\mathrm{Sb}$ atoms labeled 1, 2, and 3 in Fig. 4 are replaced successively; this yields an incorrectly $\mathrm{H} 3$-centered $\mathrm{Sb}$ trimer. For the $\mathrm{Si}_{8}$ cluster the $\mathrm{Sb}$ atoms 2, 3, and 5 [which are in an ideal $(1 \times 1)$ environment] are replaced; this yields a correctly T4-centered Sb trimer. (d) No additional Sb atoms are required to saturate any exposed Si dangling bonds. The growth always can proceed with fully saturated bonds: four neighbors for each $\mathrm{Si}$ atom and three neighbors for $\mathrm{Sb}$ atoms [except for incomplete $\mathrm{Sb}$ trimers on the $(1 \times 1)$ islands].

In conclusion, the different growth behavior for homoepitaxy on As- and Sb-covered Si (111) found in experiments can be understood on the basis of the calculations presented. (i) The calculated formation energies of single $\mathrm{Si}_{n}$ clusters on $\mathrm{Si}(111)$ : $\mathrm{As}(1 \times 1)$ confirm the result known for full layers: As trimers on top of a Si double layer are less favorable than incorporated As atoms. Double-layer growth starts already at a cluster size of four $\mathrm{Si}$ atoms. (ii) On the other hand, on $\mathrm{Si}(111): \mathrm{Sb}(1 \times 1)$ individual $\mathrm{Sb}$ trimers grow on top of a locally completed Si double layer nucleating the more favorable $(\sqrt{3} \times \sqrt{3})$ structure by single layer growth. (iii) From the energies of clusters on the $\operatorname{Si}(111): \operatorname{Sb}(\sqrt{3} \times \sqrt{3})$ terrace structure one can conclude that double-layer growth is inhibited for two reasons: (a) sufficiently large $(1 \times 1)$ cluster have to be established to support (new) T4-centered Sb trimers; (b) there is a nucleation barrier for 2nd layer trimer growth. This explains the observed $(1 \times 1)$ rim at all $\mathrm{Si}$ islands on Sb-covered $\mathrm{Si}$ (111).

We would like to thank the BMBF-Verbundprojekt 22, Halbleitergrenzflächen: Morphologie und elektronische Eigenschaften, for financial support.

[1] M. Copel, M. C. Reuter, E. Kaxiras, and R. M. Tromp, Phys. Rev. Lett. 63, 632 (1989).

[2] M. Horn-von Hoegen, F. K. LeGoues, M. Copel, M. C. Reuter, and R. M. Tromp, Phys. Rev. Lett. 67, 1130 (1991).

[3] M. Horn-von Hoegen, Z. Kristallogr. 214, 591 (1999); 214, 684 (1999).

[4] J. Falta, T. Schmidt, G. Materlik, J. Zeysing, G. Falkenberg, and R. L. Johnson, Appl. Surf. Sci. 162163, 256 (2000).

[5] B. Voigtländer, A. Zinner, T. Weber, and H. P. Bonzel, Phys. Rev. B 51, 7583 (1995).

[6] E. Kaxiras, Mater. Sci. Eng. B 30, 175 (1995).

[7] D. Kandel and E. Kaxiras, Phys. Rev. Lett. 75, 2742 (1995).

[8] E. Kaxiras, Thin Solid Films 272, 386 (1996).

[9] K. Schroeder, B. Engels, P. Richard, and S. Blügel, Phys. Rev. Lett. 80, 2873 (1998).

[10] K. Schroeder, A. Antons, R. Berger, and S. Blügel, Phys. Rev. Lett. 88, 046101 (2002).

[11] M. A. Olmstead, R. D. Bringans, R. I. G. Uhrberg, and R. Z. Bachrach, Phys. Rev. B 34, 6041 (1986).

[12] P. Martensson, G. Meyer, N. M. Amer, E. Kaxiras, and K. C. Pandey, Phys. Rev. B 42, 7230 (1990).

[13] R. Berger, S. Blügel, A. Antons, Wi. Kromen, and K. Schroeder, in Proceedings of the NIC Workshop: Molecular Dynamics on Parallel Computers (World Scientific, London, 2000), p. 185.

[14] R. Car and M. Parrinello, Phys. Rev. Lett. 55, 2471 (1985).

[15] S. H. Vosko, L. Wilk, and M. Nusair, Can. J. Phys. 58, 1200 (1980).

[16] R. O. Jones and O. Gunnarsson, Rev. Mod. Phys. 61, 689 (1989).

[17] D. Vanderbilt, Phys. Rev. B 32, 8412 (1985).

[18] L. Kleinman and D. M. Bylander, Phys. Rev. Lett. 48, 1425 (1982).

[19] N. Kosugi, J. Comput. Phys. 55, 426 (1984).

[20] P. Richard, Ph.D. thesis, RWTH Aachen, Germany, 1996, published as Report of the Research Center Jülich, Report No. Jül-3331, 1996.

[21] Broyden-Fletcher-Goldfarb-Shannoo method in R. Fletcher, Practical Methods of Optimization (John Wiley \& Sons, New York, 1987).

[22] For the larger lateral unit cell used for $(\sqrt{3} \times \sqrt{3})$ $\mathrm{Si}(111): \mathrm{Sb}$ we reduced the basis set to one $\mathbf{k}_{\|}$point and 9 Ry cutoff energy. The comparison of the energies for the smaller clusters calculated in both lateral cells shows a variation of the energy differences of $\approx 0.1 \mathrm{eV}$ for the different basis sets.

[23] K. Schroeder, A. Antons, R. Berger, and S. Blügel, Phase Transit. 75, 91 (2002). 